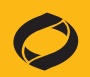

Promega

\title{
Trypsin/Lys-C protease mix for enhanced protein mass spectrometry analysis
}

\author{
Traditionally, trypsin and Lys-C proteases are used in combination to digest proteolytically \\ resistant proteins. Here we describe use of Trypsin/Lys-C Mix for general digestion needs. Working \\ simultaneously under conventional non-denaturing trypsin digestion conditions, Trypsin and Lys-C \\ enhance proteolysis and provide multiple positive effects on protein mass spectrometry analysis \\ including increased number of identified peptides and proteins, higher analytical reproducibility \\ and more accurate protein quantitation. Effectively, by supplementing trypsin with Lys-C, we create \\ improved trypsin.
}

\section{Introduction}

Trypsin is the most popular protease used in mass spectrometry because of its high proteolytic activity and cleavage specificity. However, trypsin has certain shortcomings. Digestion with trypsin is rarely complete. Additionally, tightly folded proteins resist proteolysis and many reagents used in protein preparation inhibit trypsin activity. Supplementing trypsin with Lys-C addresses these shortcomings. In 2002, Yates's group developed the sequential digestion protocol in which Lys-C supplements trypsin to overcome the proteolytic resistance of tightly folded proteins ${ }^{1}$. Here we expand the utility of Trypsin and Lys-C for general digestion needs. To avoid trypsin autoproteolysis and digestion by Lys-C during the course of the digestion reaction, we created a Trypsin/Lys-C Mix containing chemically modified Trypsin. Lys-C stability is provided by its inherent tight folding. Trypsin and Lys-C work simultaneously to digest proteins under conventional non-denaturing conditions and improve trypsin digestion without procedural changes.

\section{Enhanced proteolysis with Trypsin/Lys-C Mix}

Over $20 \%$ cleavage sites remained undigested in a yeast protein extract incubated overnight with trypsin in the study shown in Figure 1. In contrast to our expectation, the missed cleavages were not equally distributed between lysine and arginine residues. Missed lysine cleavage sites were present at disproportionally high levels compared to missed arginine sites. Analysis of missed cleavages in

Sergei Saveliev ${ }^{1}$, Mark Bratz ${ }^{1}$, Roman Zubarev ${ }^{2}$, Matt Szapacs ${ }^{3}$, Harshavardhan Budamgunta ${ }^{2}$ \& Marjeta $\mathrm{Urh}^{1}$

${ }^{1}$ Promega, Madison, Wl, ${ }^{2}$ Department of Medical Biochemistry \& Biophysics, Karolinska Institutet, Stockholm, Sweden; ${ }^{3}$ PTS-DMPK Bioanalytical Science \& Toxicokinetics, GlaxoSmithKline, King of Prussia, PA Correspondence should be addressed to S.S. (sergei.saveliev@promega.com). various trypsin digests showed that the ratio of missed lysine sites to missed arginine sites was 5:1-6:1 regardless of the sample (data not shown).

To compensate for the evident trypsin proteolytic deficiency at lysine sites, we supplemented trypsin with Lys-C protease. Lys-C cleaves lysine sites with exceptional efficiency and specificity. We digested yeast protein extract with Trypsin/Lys-C Mix overnight under conventional, non-denaturing digestion conditions. In line with our expectations, the Trypsin/Lys-C Mix reduced the number of missed lysine sites to a few percent and, thereby, improved overall proteolytic efficiency (Fig. 1).

Benefit 1: Improved protein mass spectrometry analysis Analysis of various protein samples showed that Trypsin/Lys-C Mix increased the number of identified proteins by up to $20 \%$ (Table 1)

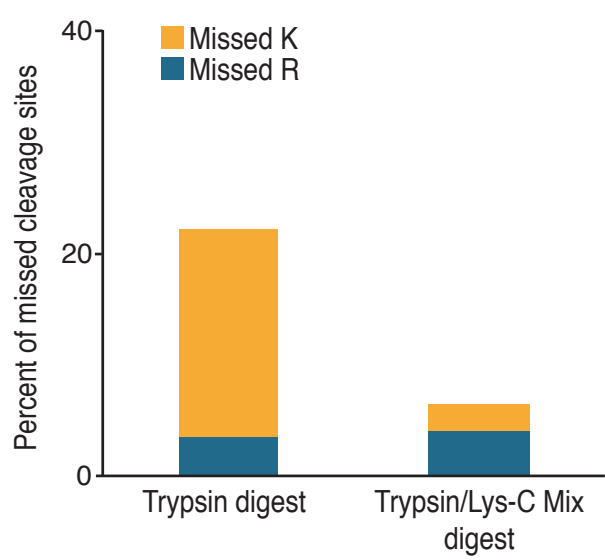

Figure 1 | Protein digestion with Trypsin/Lys-C Mix minimizes missed cleavages. Yeast total protein extract was digested overnight with trypsin or Trypsin/Lys-C Mix. The digests were analyzed using an Orbitrap Velos mass spectrometer (Thermo Fisher Scientific) 
and the number of identified peptides by up to $40 \%$ (data not shown). The proteins were digested overnight under non-denaturing conditions with either proteolytic reagent in these experiments.

Table 1 | Improved protein mass spectrometry analysis with Trypsin/Lys-C Mix. Protein samples were digested overnight side by side with trypsin or Trypsin/Lys- $C$ and analyzed with liquid chromatography-mass spectrometry.

\begin{tabular}{ccc}
\hline Sample & Protease & Identified proteins \\
\hline $\begin{array}{c}\text { Formalin-fixed } \\
\text { paraffin-embedded } \\
\text { (FFPE) skin } \\
\text { protein extract }\end{array}$ & Trypsin & 165 \\
\cline { 2 - 3 } HeLa protein extract & Trypsin/Lys-C & $182(10.3 \%$ increase) \\
\cline { 2 - 3 } & Trypsin/Lys-C & $4,108(9.3 \%$ increase $)$ \\
\hline Yeast protein extract & Trypsin & 1,252 \\
\cline { 2 - 3 } & Trypsin/Lys-C & $\begin{array}{c}1,495(19.4 \% \\
\text { increase })\end{array}$ \\
\hline
\end{tabular}

Benefit 2: Improved protein digestion reproducibility

In our model experiment, HeLa protein extract was digested in triplicate with either trypsin or Trypsin/Lys-C Mix following conventional trypsin digestion conditions for all samples. The data showed that one trypsin digest experienced an $11 \%$ drop in the number of identified proteins compared to two other trypsin digests (data not shown). In contrast, digestion with the Trypsin/Lys-C Mix was highly reproducible, as indicated by a $0.5 \%$ coefficient of variation in the number of identified proteins.

\section{Benefit 3: Improved protein quantitation}

Mass spectrometry protein quantitation methods typically use digested peptides as quantitation markers. These methods have an implicit assumption that the parent protein is completely digested. A model experiment involving serum amyloid $\mathrm{P}$ component protein showed that this assumption is incorrect when performing digestions with trypsin. Digestion with trypsin resulted in a 1.7- to 4.7-fold lower peptide peak intensity for three out of four analyzed peptides as compared to digestion with Trypsin/Lys-C Mix (data not shown). These data indicate that the Trypsin/Lys-C Mix provides conditions for more accurate protein quantitation than trypsin.

\section{Benefit 4: Tolerance to trypsin-inhibiting agents}

Many reagents used to facilitate protein extraction and preserve protein stability inhibit trypsin activity, necessitating extensive protein clean-up prior to digestion. As shown in Table 2, guanidine chloride, a reagent often used for protein solubilization and denaturation, induced abnormally high levels of missed cleavages $(44.4 \%)$ in a yeast protein extract digested with trypsin. A protease inhibitor cocktail provided even higher trypsin inhibition $(55.9 \%$ missed cleavages). When trypsin was replaced with Trypsin/Lys-C Mix, the levels of missed cleavages were restored to a more conventional level of $21-24 \%$ in both samples. These data demonstrate that Trypsin/Lys-C Mix assures efficient proteolysis in the presence of trypsin inhibiting impurities. This makes Trypsin/Lys-C Mix a protease preparation of choice in the cases where protein purity is low or when removal of protein extraction and stabilization agents is not feasible.

Table 2 | Improved Trypsin/Lys-C Mix tolerance to trypsininhibiting agents. Yeast total protein extract was digested overnight with trypsin or Trypsin/Lys-C in the presence of guanidine chloride (GuCl) or a protease inhibitor cocktail (Promega). The digests were analyzed using $Q$ Exactive mass spectrometer (Thermo Fisher Scientific).

\begin{tabular}{ccc}
\hline Agent & Protease & Missed cleavages \\
\hline \multirow{3}{*}{ GuCl (0.5 M) } & Trypsin & $\begin{array}{c}44.4 \% \\
(36.3 \% \text { missed lysine } \\
\text { sites })\end{array}$ \\
\cline { 2 - 3 } & Trypsin/Lys-C & $\begin{array}{c}21.5 \% \\
(10.3 \% \text { missed lysine } \\
\text { sites })\end{array}$ \\
\hline Protease inhibitor & & $55.9 \%$ \\
cocktail (1x) & Trypsin & $\left(\begin{array}{c}44.1 \% \text { missed lysine } \\
\text { sites })\end{array}\right.$ \\
\cline { 2 - 3 } & & $24.6 \%$ \\
& Trypsin/Lys-C & $(9.6 \%$ missed lysine \\
& & sites $)$ \\
\hline
\end{tabular}

\section{Conclusion}

Our application expands Trypsin/Lys-C utility beyond the traditional narrow use for digesting proteolytically resistant proteins. Trypsin/ Lys-C Mix works under conventional non-denaturing conditions used in general trypsin digestion protocols and improves protein digestion by eliminating the majority of missed cleavages typically occurring in trypsin digests. Trypsin/Lys-C Mix provides multiple benefits for protein mass spectrometry analysis including increasing the number of peptides and proteins identified in complex protein mixtures, improving analytical reproducibility and enabling more accurate protein quantitation. High tolerance to trypsin-inhibiting reagents used to aid protein extraction, solubilization and stability is an additional important advantage of Trypsin/Lys-C Mix. Maintaining these reagents in protein solutions is often a necessity as their removal leads to protein precipitation and other unwanted effects. By replacing trypsin with Trypsin/Lys-C Mix, adequate digestion efficiency in the presence of these reagents can be restored. The Trypsin/Lys-C Mix should be considered as a tool of choice for researchers who require higher efficiency protein mass spectrometry analysis.

\section{ACKNOWLEDGEMENTS}

We are grateful to Dr. Christopher Adams for sharing the results of Trypsin/ Lys-C Mix performance evaluation with us.

1. McDonald, W.H. et al. Comparison of three directly coupled HPLC MS/ MS strategies for identification of proteins from complex mixtures: Singledimension LC-MS/MS, 2-phase MudPIT and 3-phase MudPIT. Int. J. Mass Spectrom. 219, 245-251(2002).

This article was submitted to Nature Methods by a commercial organization and has not been peer reviewed. Nature Methods takes no responsibility for the accuracy or otherwise of the information provided. 